CERN-TH.7234/94

RAL-94-039

\title{
THE DECOUPLING THEOREM IN EFFECTIVE SCALAR FIELD THEORY
}

\author{
Richard D. Ball ${ }^{a *}$ and Robert S. Thorne ${ }^{b}$ \\ Theory Division, CERN, \\ CH-1211 Genève 23, Switzerland. ${ }^{a}$ \\ and \\ Rutherford Appleton Laboratory, \\ Chilton, Didcot, Oxon., OX11 0QX, U.K. ${ }^{b}$
}

\begin{abstract}
We consider decoupling in the context of an effective quantum field theory of two scalar fields with well separated mass scales and a $Z_{2} \times Z_{2}$ symmetry. We first prove, using Wilson's exact renormalization group equation, that the theory is renormalizable, in the same way that we showed in a previous paper that theories with a single mass scale were renormalizable. We then state and prove a decoupling theorem: at scales below the mass of the heavy particle the full theory may be approximated arbitrarily closely by an effective theory of the light particle alone, with naturalness scale the heavy particle mass. We also compare our formulation of effective field theory with the more conventional local formulation.
\end{abstract}

CERN-TH.7234/94

RAL-94-039

April 1994

* On leave of absence from a Royal Society University Research Fellowship. 
In a previous paper [1] we considered an effective scalar quantum field theory containing a single propagating scalar particle with a $Z_{2}$ global symmetry. We constructed a regulated classical action for this theory which is expandable as a convergent infinite series of local terms, and is characterized by some such naturalness scale, $\Lambda_{0}$, at which all the coupling constants in the action are of order one when expressed in units of $\Lambda_{0}$. The particle mass $m$ is assumed to be finetuned so that $m \ll \Lambda_{0}$, so that we can consider scattering amplitudes at energy scales $E \ll \Lambda_{0}$. We were then able to show that not only is the S-matrix of the theory well-defined, unitary and causal at all scales, but that the theory is perturbatively renormalizable, in the sense that these low-energy scattering amplitudes can be expressed with great accuracy in terms of only a finite number of physically relevant parameters. Technically this separation of scales was achieved through a systematic exploitation of Wilson's exact renormalization group equation $[2,3]$. All the results may be readily generalized to theories with many scalar or spin-half particles, possibly with linearly realized global symmetries, provided all the particles have masses $m_{i}$ of the same order of magnitude.

Now as the energy scale $E$ at which the physics of the theory is being probed is increased, we were able to show further that the degree of predictivity of the theory can be maintained by including more and more measurable parameters (by fixing more and more low energy renormalization conditions on couplings). We could also derive Weinberg-style bounds on the growth with $E$ of Euclidean Green's functions [4]. For energies of order $\Lambda_{0}$ we need formally an infinite number of parameters and have no real predictive power beyond that offered by S-matrix theory; indeed it was possible to show that the effective field theory and S-matrix theory are formally equivalent.

As physicists we are sufficiently optimistic to hope that the naturalness scale $\Lambda_{0}$ will be the scale at which we will find some sort of new physics which will serve to substantially restrict the infinite number of possible theories to some more manageable finite subset, so as to regain a reasonably predictive theory over a new range of energy scales. However, the effective theory as considered so far is phenomenologically neutral, in the sense that by construction it is completely independent of the form this new physics may take, provided only that it be consistent with the basic principles of special relativity and quantum mechanics. Indeed, this is why there are so many possible effective theories of even a single scalar particle. So at $\Lambda_{0}$ it is desirable to put forward additional assumptions. Here we will consider what is probably the simplest such assumption: that a new scalar particle of mass $M$ of the order of $\Lambda_{0}$ is discovered, and that the naturalness scale of the new theory can then be pushed, by further fine tuning, to even higher scales. We are thus led to consider the following simple paradigm; a theory containing two scalar particles with significantly different masses $m \ll M \ll \Lambda_{0}$, in which we can then determine the dependence of the physics at various scales depends on the ratio of the masses of the particles. In particular, we will be able to see exactly how the heavy particle 'decouples' from the low energy effective theory containing only the light particle.

Decoupling in local quantum field theories was first investigated in the early 1970's, with the development of a decoupling theorem [5] which stated that if we have a local quantum field theory 
in which some particles have masses $M$ very large compared to the others then Green's functions for light particle processes at energies $E \ll M$ are the same as those in the local theory obtained by simply omitting the heavy particles, up to corrections of inverse powers of the heavy mass. The only effect of the heavy particles is thus to renormalize the masses and relevant couplings in the light particle theory. Rigorous proofs [6] of this decoupling theorem using the BPHZ subtraction formalism took some time to develop, however, essentially because in this formalism one attempts to deal with all the different scales, including of course the cut-off $\Lambda_{0}$, at the same time. If the light particles are massless, there are further complications, and extensions of the decoupling theorem to include the leading order $E^{2} / M^{2}$ corrections [7] become increasingly awkward to deal with satisfactorily.

We will find here that on the contrary, since exact renormalization group techniques are very good at separating the physics at different scales, it is relatively straightforward to use them to prove an extended decoupling theorem which can treat corrections to the light particle effective theory of arbitrarily high orders in $E^{2} / M^{2}$. In effect the theorem reduces to a statement of complex analysis: the light particle vertex functions of the full theory, which have Taylor expansions with radius of convergence $M$, may be approximated to arbitrary accuracy within their circle of convergence by the regular vertex functions of the light theory.

This relatively straightforward interpretation of decoupling is also due in part to our particular formulation of effective field theory in [1]. In the more conventional approach to effective theories which was in fact motivated in part by precisely the sort of decoupling situation discussed here[8], the effective theory of the light particle is always considered only in the strictly local limit in which the cut-off is removed. This makes it difficult to entertain the possibility of an effective theory which is not tied in some way to an underlying local 'fundamental' theory. For us the light particle theory is simply an effective theory with naturalness scale of order $M$, to be (perhaps) superseded at scales of order $M$ by another effective theory with naturalness scale $\Lambda_{0} \gg M$, to which it is matched.

Once we have defined precisely the full theory we are working with, with the three wellseparated scales $m \ll M \ll \Lambda_{0}$, our first step will be to prove the boundedness and convergence of this theory $(\S 2)$. Once we have done this it will be relatively easy to prove in $\S 3$ the conventional form of the decoupling theorem. In $\S 4$ we will then show how we can systematically improve this decoupling theorem to make the predictions of the low energy theory arbitrarily accurate at a given low energy scale, and finally in $\S 5$ we discuss the relation between our formulation of effective theories and the more conventional one.

\section{Defining the Theory}

We will prove the decoupling theorem for the simplest case, i.e. a single light scalar particle described by the field $\phi$, and a single heavy scalar particle described by the field $\Phi$, where the theory is invariant under a $Z_{2} \times Z_{2}$ global symmetry, under which the fields transform as $\phi \rightarrow-\phi$, $\Phi \rightarrow-\Phi$. 
We must first define the theory containing these two particles. As explained in [1], the effective quantum field theory is defined by its field content, global symmetries, the form of the propagators, and a full set of boundary conditions on the renormalization group flow for the interaction part of the Lagrangian. As in [1] we separate the Euclidean classical action into a quadratic part and an interaction:

$$
S\left[\phi, \Phi ; \Lambda_{0}\right]=\frac{1}{2}\left(\phi, P_{\Lambda}^{-1} \phi\right)+\frac{1}{2}\left(\Phi, \widetilde{P}_{\Lambda}^{-1} \Phi\right)+S_{\mathrm{int}}\left[\phi, \Phi ; \Lambda_{0}\right]
$$

The propagators are defined by

$$
\begin{aligned}
& P_{\Lambda}(p)=\frac{K\left(\left(p^{2}+m^{2}\right) / \Lambda^{2}\right)}{\left(p^{2}+m^{2}\right)}=\frac{K_{\Lambda}(p)}{\left(p^{2}+m^{2}\right)}, \\
& \widetilde{P}_{\Lambda}(p)=\frac{\widetilde{K}\left(\left(p^{2}+M^{2}\right) / \Lambda^{2}\right)}{\left(p^{2}+M^{2}\right),}=\frac{\widetilde{K}_{\Lambda}(p)}{\left(p^{2}+M^{2}\right)},
\end{aligned}
$$

where $K(x), \widetilde{K}(x)$ are regular regulating functions with the same properties as those used in [1]. ${ }^{1}$ The interaction part of the action is defined to be power series in the fields $\phi$ and $\Phi$; because of the assumed $Z_{2} \times Z_{2}$ global symmetry only even powers appear. For perturbative purposes it is also assumed to be a formal power series in three small expansion parameters, $g_{m}, g_{M}, g_{m M}$, beginning at first order in at least one of these $\left(g_{m}, g_{M}\right.$ and $g_{m M}$ will normally be related to the coupling constants of the theory). So

$$
\begin{aligned}
S_{\mathrm{int}}[\phi, \Phi ; \Lambda] \equiv \sum_{m, n=0}^{\infty} \sum_{r, s, t=0}^{\infty} \frac{g_{m}^{r} g_{M}^{s} g_{m M}^{t}}{(2(m+n)) !} \int \frac{d^{4} p_{1} \cdots d^{4} p_{2(m+n)}}{(2 \pi)^{4(2(m+n)-1)}} & \\
& V_{2 m, 2 n}^{r, s, t}\left(p_{1}, \ldots, p_{2(m+n)} ; \Lambda\right) \delta^{4}\left(\sum_{i=1}^{2(m+n)} p_{i}\right) \phi_{p_{1}} \cdots \phi_{p_{2 m}} \Phi_{p_{2 m+1}} \cdots \Phi_{p_{2(m+n)}},
\end{aligned}
$$

where $V_{2 m, 2 n}^{r, s, t}\left(p_{1}, \ldots, p_{2(m+n)} ; \Lambda\right) \equiv V_{2 m, 2 n}^{r, s, t}\left(p_{i} ; \Lambda\right)$ is the value, at order $r$ in $g_{m}$, order $s$ in $g_{M}$ and order $t$ in $g_{m M}$, of the vertex in the effective action defined at $\Lambda$ with $2 m \phi$-legs and $2 n \Phi$-legs; $V_{2 m, 2 n}^{r, s, t}\left(p_{i} ; \Lambda\right) \equiv 0$ if $r=s=t=0$ or if $m=n=0$.

We define a complete set of boundary conditions on the renormalization group flow by setting all of the irrelevant couplings at $\Lambda_{0}$, the naturalness scale for the full theory, equal to zero (this assumption will be relaxed in $\S 2.2$ below),

$$
\partial_{p}^{j} V_{2 m, 2 n}^{r, s, t}\left(\Lambda_{0}\right)=0 \quad 2(m+n)+j>4,
$$

and choosing the relevant couplings at $\Lambda=0$ to be equal to $\Lambda_{0}$-independent constants (at each order in perturbation theory):

$$
\begin{array}{cl}
\lim _{\Lambda \rightarrow 0} V_{2,0}^{r, s, t}\left(P_{0},-P_{0} ; \Lambda\right)=\Lambda_{m}^{2} \widehat{\lambda}_{1}^{r, s, t}, & \lim _{\Lambda \rightarrow 0}\left[\left.\partial_{p_{\mu}} \partial_{p_{\nu}} V_{2,0}^{r, s, t}(p,-p ; \Lambda)\right|_{p=P_{0}}\right]_{\delta_{\mu \nu}}=\widehat{\lambda}_{2}^{r, s, t}, \\
\lim _{\Lambda \rightarrow 0} V_{0,2}^{r, s, t}\left(\widetilde{P_{0}},-\widetilde{P}_{0} ; \Lambda\right)=\Lambda_{M}^{2} \widehat{\lambda}_{4}^{r, s, t}, & \lim _{\Lambda \rightarrow 0}\left[\left.\partial_{p_{\mu}} \partial_{p_{\nu}} V_{0,2}^{r, s, t}(p,-p ; \Lambda)\right|_{p=\widetilde{P}_{0}}\right]_{\delta_{\mu \nu}}=\widehat{\lambda}_{5}^{r, s, t}, \\
V_{4,0}^{r, s, t}\left(P_{1}, P_{2}, P_{3}, P_{4} ; 0\right)=\widehat{\lambda}_{3}^{r, s, t}, & V_{0,4}^{r, s, t}\left(\widetilde{P}_{1}, \widetilde{P}_{2}, \widetilde{P}_{3}, \widetilde{P}_{4} ; 0\right)=\widehat{\lambda}_{6}^{r, s, t}, \quad V_{2,2}^{r, s, t}\left(P_{5}, P_{6}, \widetilde{P}_{5}, \widetilde{P}_{6} ; 0\right)=\widehat{\lambda}_{7}^{r, s, t},
\end{array}
$$

\footnotetext{
${ }^{1}$ In practice it would be simpler to take $K=\widetilde{K}$, but this is not necessary.
} 
where $\sum_{1}^{4} P_{i}=\sum_{1}^{4} \widetilde{P}_{i}=\sum_{5,6}\left(P_{i}+\widetilde{P}_{i}\right)=0$. By convention the series expansions for those couplings corresponding to vertices with $m=0$ are such that $r=0, t=0$, those for $n=0$ have $s=0, t=0$ and for both $m \neq 0$ and $n \neq 0, r=s=0$. We choose the external momenta $P_{i}$ with magnitudes of order $\Lambda_{m}$, where $\Lambda_{m} \sim m$, for the legs of vertices corresponding to $\phi$ fields, and the external momenta $\widetilde{P}_{i}$ with magnitudes of order $\Lambda_{M}$, where $\Lambda_{M} \sim M$, for the legs of vertices corresponding to $\Phi$ fields; the only vertex where we have a mixture of magnitudes of momenta is then the vertex with two $\phi$ legs and two $\Phi$ legs, where we have two momenta $\sim \Lambda_{m}$ and two momenta $\sim \Lambda_{M}$. In this way the renormalization conditions (1.5) may be readily continued on-shell, where they would correspond to physically observable masses and couplings. Setting all the renormalization conditions for momenta of the same order of magnitude would be artificial, in the sense that the relation between the renormalized 'masses' and 'couplings' $\lambda_{i} \equiv \sum_{r, s, t} \widehat{\lambda}_{i}^{r, s, t}$ and the physical parameters could (and in general will) involve large renormalization factors, depending on the ratio $\Lambda_{M} / \Lambda_{m} \sim M / m$.

The quantum theory of these two massive particles is then defined as in [1] by reducing $\Lambda$ from the naturalness scale $\Lambda_{0}$ through the two mass scales $\Lambda_{M}$ and $\Lambda_{m}$ down to zero, while keeping the connected amputated Green's functions $\widetilde{G}_{2 m, 2 n}^{c}$; the interaction $S_{\text {int }}[\phi, \Phi ; \Lambda]$ must the satisfy the exact renormalization group equation

$$
\frac{\partial S_{\mathrm{int}}}{\partial \Lambda}=\frac{1}{2} \int \frac{d^{4} p}{(2 \pi)^{4}}\left\{\frac{\partial P_{\Lambda}}{\partial \Lambda}\left[\frac{\delta S_{\mathrm{int}}}{\delta \phi_{p}} \frac{\delta S_{\mathrm{int}}}{\delta \phi_{-p}}-\frac{\delta^{2} S_{\mathrm{int}}}{\delta \phi_{p} \delta \phi_{-p}}\right]+\frac{\partial \widetilde{P}_{\Lambda}}{\partial \Lambda}\left[\frac{\delta S_{\mathrm{int}}}{\delta \Phi_{p}} \frac{\delta S_{\mathrm{int}}}{\delta \Phi_{-p}}-\frac{\delta^{2} S_{\mathrm{int}}}{\delta \Phi_{p} \delta \Phi_{-p}}\right]\right\} .
$$

The connected amputated Green's functions may then be read off directly from $S_{\text {int }}[\phi, \Phi ; 0]$ :

$$
\left.\widetilde{G}_{2 m, 2 n}^{c}\left(p_{1}, \ldots, p_{2(m+n)}\right) \equiv \prod_{i=1}^{2 m}\left(-\frac{\delta}{\delta \phi_{p_{i}}}\right) \prod_{j=1}^{2 n}\left(-\frac{\delta}{\delta \Phi_{p_{j}}}\right) S_{\mathrm{int}}[\phi, \Phi ; 0]\right|_{\phi=\Phi=0}
$$

\section{Renormalizability.}

We now consider the theory described in the previous section when all three scales are well separated: $\Lambda_{m} \ll \Lambda_{M} \ll \Lambda_{0}$. We wish to derive bounds on Green's functions analogous to those proved in [1] for the theory with only a single mass scale (i.e. with $\Lambda_{m} \sim \Lambda_{M} \ll \Lambda_{0}$ ), and then show further that the Green's functions are convergent (independent of $\Lambda_{0}$ up to power suppressed corrections) and universal (independent of the boundary conditions at $\Lambda_{0}$ on irrelevant vertices, again up to power suppressed corrections). We will then have proven that the theory is 'renormalizable', and can proceed to an investigation of decoupling.

\subsection{Boundedness.}

In order to derive appropriate bounds, we use similar methods to those in used in [1] for lemmas 1-3; we first derive bounds on the vertices and their derivatives using the flow equation 
(1.6), and then proceed by induction in the order of the vertices and the number of their legs. The norm on the vertices is now defined as

$$
\left\|V_{2 m, 2 n}\right\|_{\Lambda_{1}, \Lambda_{2}} \equiv \max _{\left\{p_{1}, \ldots, p_{2(m+n)}\right\}}\left[\prod_{i=1}^{2 m}\left[K_{\Lambda_{1}}\left(p_{i}\right)\right]^{1 / 4} \prod_{j=1}^{2 n}\left[\widetilde{K}_{\Lambda_{2}}\left(p_{j}\right)\right]^{1 / 4}\left|V\left(p_{1}, \ldots, p_{2(m+n)} ; \Lambda\right)\right|\right] .
$$

If a vertex does not have legs corresponding to one of the fields (i.e. if either $m$ or $n$ is zero) we will omit the superscript referring to the damping factors attached to these legs.

The flow equation for the vertices is obtained by substituting (1.3) into (1.6) and we can bound the $j_{t h}$ momentum derivative of the left hand side to give

$$
\begin{aligned}
& \left\|\frac{\partial}{\partial \Lambda}\left(\partial_{p}^{j} V_{2 m, 2 n}^{r, s, t}(\Lambda)\right)\right\|_{\Lambda, \Lambda} \leq \Lambda\left\|\partial_{p}^{j} V_{2 m+2,2 n}^{r, s, t}(\Lambda)\right\|_{\Lambda, \Lambda}+\Lambda\left\|\partial_{p}^{j} V_{2 m, 2 n+2}^{r, s, t}(\Lambda)\right\|_{\Lambda, \Lambda} \\
& +\sum_{r^{\prime}=1}^{r-1} \sum_{s^{\prime}=1}^{s-1} \sum_{t^{\prime}=1}^{t-1} \sum_{j_{i} ; j_{1}+j_{2}+j_{3}=j}\left[\sum_{l=1}^{m} \sum_{k=0}^{n} \Lambda^{-3-j_{1}}\left\|\partial_{p}^{j_{2}} V_{2 l, 2 k}^{r^{\prime}, s^{\prime}, t^{\prime}}(\Lambda)\right\|_{\Lambda, \Lambda} \cdot\left\|\partial_{p}^{j_{3}} V_{2 m+2-2 l, 2 n-2 k}^{r-r^{\prime}, s-s^{\prime}, t-t^{\prime}}(\Lambda)\right\|_{\Lambda, \Lambda}\right. \\
& \left.\quad+\sum_{l=0}^{m} \sum_{k=1}^{n} \Lambda^{-3-j_{1}}\left\|\partial_{p}^{j_{2}} V_{2 l, 2 k}^{r^{\prime}, s^{\prime}, t^{\prime}}(\Lambda)\right\|_{\Lambda, \Lambda} \cdot\left\|\partial_{p}^{j_{3}} V_{2 m-2 l, 2 n-2 k+2}^{r-r^{\prime}, s-s^{\prime}, t-t^{\prime}}(\Lambda)\right\|_{\Lambda, \Lambda}\right],
\end{aligned}
$$

for $\Lambda \in\left[\Lambda_{M}, \Lambda_{0}\right]$. For $\Lambda \in\left[0, \Lambda_{M}\right]$ we find instead

$$
\begin{aligned}
& \left\|\frac{\partial}{\partial \Lambda}\left(\partial_{p}^{j} V_{2 m, 2 n}^{r, s, t}(\Lambda)\right)\right\|_{\Lambda^{\prime}, \Lambda_{M}} \leq \Lambda\left\|\partial_{p}^{j} V_{2 m+2,2 n}^{r, s, t}(\Lambda)\right\|_{\Lambda^{\prime}, \Lambda_{M}}+\Lambda\left\|\partial_{p}^{j} V_{2 m, 2 n+2}^{r, s, t}(\Lambda)\right\|_{\Lambda^{\prime}, \Lambda_{M}} \\
& +\sum_{r^{\prime}=1}^{r-1} \sum_{s^{\prime}=1}^{s-1} \sum_{t^{\prime}=1}^{t-1} \sum_{j_{i} ; j_{1}+j_{2}+j_{3}=j}\left[\sum_{l=1}^{m} \sum_{k=0}^{n} \Lambda^{-3-j_{1}}\left\|\partial_{p}^{j_{2}} V_{2 l, 2 k}^{r^{\prime}, s^{\prime}, t^{\prime}}(\Lambda)\right\|_{\Lambda^{\prime}, \Lambda_{M}} \cdot\left\|\partial_{p}^{j_{3}} V_{2 m+2-2 l, 2 n-2 k}^{r-r^{\prime}, s-s^{\prime}, t-t^{\prime}}(\Lambda)\right\|_{\Lambda^{\prime}, \Lambda_{M}}\right. \\
& \left.\quad+\sum_{l=0}^{m} \sum_{k=1}^{n} \Lambda^{-3-j_{1}} K^{1 / 2}\left(\frac{M^{2}}{\Lambda^{2}}\right)\left\|\partial_{p}^{j_{2}} V_{2 l, 2 k}^{r^{\prime}, s^{\prime}, t^{\prime}}(\Lambda)\right\|_{\Lambda^{\prime}, \Lambda_{M}} \cdot\left\|\partial_{p}^{j_{3}} V_{2 m-2 l, 2 n-2 k+2}^{r-r^{\prime}, s-s^{\prime}, t-t^{\prime}}(\Lambda)\right\|_{\Lambda^{\prime}, \Lambda_{M}}\right],
\end{aligned}
$$

where $\Lambda^{\prime}=\max \left(\Lambda, \Lambda_{m}\right)$ : for $\Lambda \in\left[\Lambda_{m}, \Lambda_{M}\right]$ we take $\Lambda^{\prime}=\Lambda$, while for $\Lambda \in\left[0, \Lambda_{m}\right]$ we take $\Lambda^{\prime}=\Lambda_{m}$. We have explicitly retained the factor of $K^{1 / 2}\left(M^{2} / \Lambda^{2}\right)$ arising from the $\Lambda$-derivative of the propagator in the last term since it will turn out to be be important for part of the argument.

We may then prove the following bounds: ${ }^{2}$

\section{Lemma 8:}

i) For $\Lambda \in\left[\Lambda_{M}, \Lambda_{0}\right]$, and for all $m, n, j, r, s$ and $t$,

$$
\left\|\partial_{p}^{j} V_{2 m, 2 n}^{r, s, t}(\Lambda)\right\|_{\Lambda, \Lambda} \leq \Lambda^{4-2 m-2 n-j}\left(P \log \left(\frac{\Lambda}{\Lambda_{m}}\right)+\frac{\Lambda}{\Lambda_{0}} P \log \left(\frac{\Lambda_{0}}{\Lambda_{m}}\right)\right) .
$$

ii) For $\Lambda \in\left[\Lambda_{m}, \Lambda_{M}\right]$, and for all $m, j, r, s$ and $t$, except $m=0, n=1$ and $j=0,1$,

$$
\left\|\partial_{p}^{j} V_{2 m, 2 n}^{r, s, t}(\Lambda)\right\|_{\Lambda, \Lambda_{M}} \leq \begin{cases}\Lambda^{4-2 m-2 n-j} P \log \left(\frac{\Lambda_{M}}{\Lambda_{m}}\right), & \text { if } n>0 \\ \Lambda^{4-2 m-j} P \log \left(\frac{\Lambda}{\Lambda_{m}}\right), & \text { if } n=0\end{cases}
$$

\footnotetext{
2 To avoid confusion we number the lemmas in [1], [4] and the present paper consecutively.
} 
iii) For $\Lambda \in\left[0, \Lambda_{m}\right]$, and for all $m, j, r, s$ and $t$, except for $m=0, n=1$, and $j=0,1$,

$$
\left\|\partial_{p}^{j} V_{2 m, 2 n}^{r, s, t}(\Lambda)\right\|_{\Lambda_{m}, \Lambda_{M}} \leq \begin{cases}\Lambda_{m}^{4-2 m-2 n-j} P \log \left(\frac{\Lambda_{M}}{\Lambda_{m}}\right), & \text { if } n>0 \\ \Lambda_{m}^{4-2 m-j}, & \text { if } n=0 .\end{cases}
$$

iv) For the special case $m=0, n=1, j=0,1$,

$$
\left\|\partial_{p}^{j} V_{0,2}^{r, s, t}(\Lambda)\right\|_{\Lambda_{M}} \leq \Lambda_{M}^{2-j} P \log \left(\frac{\Lambda_{M}}{\Lambda_{m}}\right)
$$

for all $\Lambda \in\left[0, \Lambda_{M}\right]{ }^{3}$

The lemma is proven using essentially the same inductive method as we used for lemma 1 in [1]; the fact that we have more than one expansion parameter causes no problems, since we may simply perform the inductive arguments successively in the parameters. We thus assume that the lemma is true at order $r-1, s-1, t-1$ in the expansion parameters and order $m+1, n+1$ in the number of legs, and then investigate whether it is true at order $r, s, t$ and $m, n$; the vanishing of the vertices for large enough $m$ and $n$ for any given order in $r, s, t$, and the fact that the bounds are trivially true when $r=s=t=0$ then completes the induction. Complications arise however because we had to set the renormalization conditions (1.5) at momenta consistent with two different scales, $\Lambda_{m}$ and $\Lambda_{M}$. This causes no real difficulties if we are careful about the order in which we bound the vertices. In fact each inductive step now proceeds in six distinct steps: a) we prove i) for the irrelevant vertices; b) ii) for the irrelevant vertices; c) iii) for the irrelevant vertices; d) iii) for relevant vertices involving only light fields, and ii), iii) and iv) those involving heavy fields; e) ii) for the relevant vertices involving only light fields; and finally f) i) for the relevant vertices.

a) We first consider irrelevant vertices for $\Lambda \in\left[\Lambda_{M}, \Lambda_{0}\right]$. The flow equations are perfectly consistent with i), so integrating from $\Lambda \geq \Lambda_{M}$ up to $\Lambda_{0}$ and using the fact that the irrelevant vertices at $\Lambda_{0}$ are equal to zero, just as in part a) of the proof of lemma 1 , i) is seen to be true at the next order.

b) We now investigate the irrelevant vertices for $\Lambda \in\left[\Lambda_{m}, \Lambda_{M}\right]$. If we integrate the left hand side of (2.3) from $\Lambda$ up to $\Lambda_{M}$, where $\Lambda_{m} \leq \Lambda \leq \Lambda_{M}$, we obtain the equality

$$
\partial_{p}^{j} V_{2 m, 2 n}^{r, s, t}(\Lambda)-\partial_{p}^{j} V_{2 m, 2 n}^{r, s, t}\left(\Lambda_{M}\right)=\int_{\Lambda}^{\Lambda_{M}} d \Lambda^{\prime} \frac{\partial}{\partial \Lambda^{\prime}}\left(\partial_{p}^{j} V_{2 m, 2 n}^{r, s, t}\left(\Lambda^{\prime}\right)\right)
$$

3 The necessity for this exceptional case is easy to understand if we remember that $V_{0,2}^{r, s, t}(\Lambda)$ are the corrections to the mass term of the effective Lagrangian for the heavy field. As discussed in $\S 3.2$ of [1], we would naively expect these corrections to be of order $\Lambda_{0}^{2}$ for all $\Lambda$, but the fine-tuned renormalization condition (1.5) which specifies the mass corrections to be of order $\Lambda_{M}^{2}$ at each order in the two point Green's function, forces them to be of order $\Lambda^{2}$ as we flow down from $\Lambda_{0}$ to $\Lambda_{M}$. However as we flow down further the corrections now behave as we would actually expect from the naive dimensional argument, and are thus roughly the same for all $\Lambda \leq \Lambda_{M}$. This is in contrast with the mass corrections on the light particle, which are forced to be 'unnaturally' small by their renormalization condition, so that $V_{2,0}^{r, s, t}(\Lambda)$ satisfies ii) and iii). 
which is easily turned into the inequality

$$
\left\|\partial_{p}^{j} V_{2 m, 2 n}^{r, s, t}(\Lambda)\right\|_{\Lambda, \Lambda_{M}} \leq\left\|\partial_{p}^{j} V_{2 m, 2 n}^{r, s, t}\left(\Lambda_{M}\right)\right\|_{\Lambda_{M}, \Lambda_{M}}+\int_{\Lambda}^{\Lambda_{M}} d \Lambda^{\prime}\left\|\frac{\partial}{\partial \Lambda^{\prime}}\left(\partial_{p}^{j} V_{2 m, 2 n}^{r, s, t}\left(\Lambda^{\prime}\right)\right)\right\|_{\Lambda^{\prime}, \Lambda_{M}}
$$

We first consider the vertices with $n>0$, in which case all vertices on the right-hand side of (2.3) also have $n>0$. Simply by evaluating i) at $\Lambda=\Lambda_{M}$, we know that the first term on the right-hand side of (2.9) is $\leq \Lambda_{M}^{4-2 m-2 n-j} P \log \left(\frac{\Lambda_{M}}{\Lambda_{m}}\right)$. However, we might think that the bound on $V_{0,2}^{r, s, t}(\Lambda)$ and its first momentum derivative would cause the second term on the right of (2.9) to be inconsistent with (ii). It actually causes no problem since, as we see from (2.3), it always appears along with the factor $K^{1 / 2}\left(M^{2} / \Lambda^{2}\right) . M \sim \Lambda_{M}$, so as far as the right hand side of the flow equation is concerned the bound on $V_{0,2}^{r, s, t}(\Lambda)$ is

$$
\left\|V_{0,2}^{r, s, t}(\Lambda)\right\|_{\Lambda_{M}} \leq \Lambda_{M}^{2} K^{1 / 2}\left(\frac{\Lambda_{M}^{2}}{\Lambda^{2}}\right) P \log \left(\frac{\Lambda_{M}}{\Lambda_{m}}\right) \leq \Lambda^{2} P \log \left(\frac{\Lambda_{M}}{\Lambda_{m}}\right)
$$

since $x K^{1 / 2}(x) \leq c$. A similar result is obviously true for the first momentum derivative of $V_{0,2}^{r, s, t}(\Lambda)$. We can therefore say that

$$
\left\|\frac{\partial}{\partial \Lambda}\left(\partial_{p}^{j} V_{2 m, 2 n}^{r, s, t}(\Lambda)\right)\right\|_{\Lambda, \Lambda_{M}} \leq \Lambda^{3-2 m-2 n-j} P \log \left(\frac{\Lambda_{M}}{\Lambda_{m}}\right)
$$

for $n>0$. Thus, the term on the far right in (2.9) is perfectly consistent with ii), and (2.9) coupled with the induction hypothesis and the bounds already obtained on the vertices at $\Lambda_{M}$ immediately lead to the verification of ii) for all the irrelevant vertices with $n>0$.

We can now prove ii) for the irrelevant vertices with $n=0$. The only obstacle to proving this bound using (2.9) comes from the term on the right-hand side of (2.3) containing $\left\|V_{2 m, 2}^{r, s, t}(\Lambda)\right\|_{\Lambda, \Lambda_{M}}$. From above we already know that when $\Lambda$ is between $\Lambda_{m}$ and $\Lambda_{M}$, $\left\|V_{2 m, 2}^{r, s, t}(\Lambda)\right\|_{\Lambda, \Lambda_{M}} \leq \Lambda^{2-2 m-j} P \log \left(\Lambda_{M} / \Lambda_{m}\right)$ for all vertices which could appear on the righthand side of (2.3). Thus, within this range of $\Lambda$, the problematic term is bounded by $\Lambda^{3-2 m-j} K^{1 / 2}\left(\frac{\Lambda_{M}^{2}}{\Lambda^{2}}\right) P \log \left(\frac{\Lambda_{M}}{\Lambda_{m}}\right)$. To show that this term in the flow equation is consistent with the induction argument needed to prove ii), we may use the inequality

$$
K^{1 / 2}\left(\frac{\Lambda_{M}}{\Lambda}\right) P\left[\log \left(\frac{\Lambda_{M}}{\Lambda}\right)+\log \left(\frac{\Lambda}{\Lambda_{m}}\right)\right] \leq P \log \left(\frac{\Lambda}{\Lambda_{m}}\right)
$$

for all $\Lambda \in\left[\Lambda_{m}, \Lambda_{M}\right]$. By substituting this bound into the right hand side of (2.3) we obtain

$$
\left\|\frac{\partial}{\partial \Lambda}\left(\partial_{p}^{j} V_{2 m, 0}^{r, s, t}(\Lambda)\right)\right\|_{\Lambda, \Lambda_{M}} \leq \Lambda^{3-2 m-j}\left(P \log \left(\frac{\Lambda}{\Lambda_{m}}\right)+\frac{\Lambda}{\Lambda_{0}} P \log \left(\frac{\Lambda_{0}}{\Lambda_{m}}\right)\right)
$$

for all $\Lambda \in\left[\Lambda_{m}, \Lambda_{M}\right]$. The induction argument now goes through as normal and the irrelevant vertices with $\phi$ legs only satisfy ii). 
c) The proofs of iii) for the irrelevant vertices follow much the same lines as those of ii). In both cases we integrate the left-hand side of (2.3) from $\Lambda$ up to $\Lambda_{m}$. The boundary conditions obtained from ii), evaluated at $\Lambda=\Lambda_{m}$, are consistent with iii). We therefore only have to be concerned with the right-hand side of (2.3). We first consider $n>0$. We may substitute $K^{1 / 2}\left(\frac{M^{2}}{\Lambda^{2}}\right) \Lambda^{-3-j_{1}}$ in the last term on the right-hand side by $\Lambda_{m}^{-3-j_{1}}$ (we could obviously do better than this if we wanted, but this result is sufficient to prove the lemma), or for the two point vertex for the heavy field, replace $K^{1 / 2}\left(M^{2} / \Lambda^{2}\right) \Lambda^{-3-j_{1}} \Lambda_{M}^{2}$ by $\Lambda^{1-j_{1}}$. Once we have done this we may write

$$
\left\|\frac{\partial}{\partial \Lambda}\left(\partial_{p}^{j} V_{2 m, 2 n}^{r, s, t}(\Lambda)\right)\right\|_{\Lambda_{m}, \Lambda_{M}} \leq \Lambda_{m}^{3-2 m-2 n-j} P \log \left(\frac{\Lambda_{M}}{\Lambda_{m}}\right),
$$

for $n>0$ and all $\Lambda \in\left[0, \Lambda_{m}\right]$. With this result the proof of iii) immediately goes through. For $n=0$, it is again only the term containing $\left\|V_{2 m, 2}^{r, s, t}(\Lambda)\right\|_{\Lambda_{m}, \Lambda_{M}}$ that causes a potential obstruction. We easily see that $K^{1 / 2}\left(\Lambda_{M}^{2} / \Lambda^{2}\right) P \log \left(\frac{\Lambda_{M}}{\Lambda_{m}}\right) \leq c$ for $\Lambda \leq \Lambda_{m}$, and so

$$
\left\|\frac{\partial}{\partial \Lambda}\left(\partial_{p}^{j} V_{2 m, 0}^{r, s, t}(\Lambda)\right)\right\|_{\Lambda_{m}, \Lambda_{M}} \leq \Lambda_{m}^{3-2 m-j}
$$

for all $\Lambda \in\left[0, \Lambda_{m}\right]$. The proof of iii) for $n=0$ can now also go through without obstruction.

d) It is now possible to show that the lemma is also true for the relevant vertices. We start with the four-point vertex with $\Phi$ legs, and prove ii) and iii). In order to do this we use the renormalization conditions (1.5) at $\Lambda=0$ to provide bounds on the relevant coupling constant, i.e the vertex for the momenta $\widetilde{P}_{i}$ at which the renormalization conditions are set, at $\Lambda_{M}$. We therefore use (2.3), and the bounds already obtained for the vertices at lower order in the expansion parameters or at equal order in the expansion parameters, but with greater numbers of legs, to verify (2.11) and (2.13), and write

$$
\left|\frac{\partial}{\partial \Lambda} V_{0,4}^{r, s, t}\left(\widetilde{P}_{i} ; \Lambda\right)\right| \leq \Lambda^{-1} P \log \left(\frac{\Lambda_{M}}{\Lambda_{m}}\right),
$$

for $\Lambda \in\left[\Lambda_{m}, \Lambda_{R}\right]$, and the same if we replace $\Lambda^{-1}$ by $\Lambda_{m}^{-1}$ for $\Lambda \in\left[0, \Lambda_{m}\right]$. But,

$$
\left|V_{4}^{r, s, t}\left(\widetilde{P}_{i} ; \Lambda_{R}\right)\right| \leq\left|V_{0,4}^{r}\left(\widetilde{P}_{i} ; 0\right)\right|+\int_{0}^{\Lambda_{R}} d \Lambda^{\prime}\left|\frac{\partial}{\partial \Lambda^{\prime}} V_{0,4}^{r, s, t}\left(\widetilde{P}_{i} ; \Lambda^{\prime}\right)\right| .
$$

So, using the renormalization condition on $V_{0,4}^{r, s, t}\left(\widetilde{P}_{i} ; 0\right)$, and splitting the integral into two, one from $\Lambda=0$ to $\Lambda_{m}$ and the other from $\Lambda_{m}$ to $\Lambda_{M}$, we find that

$$
\left|V_{0,4}^{r, s, t}\left(\widetilde{P}_{i} ; \Lambda_{M}\right)\right| \leq P \log \left(\frac{\Lambda_{R}}{\Lambda_{m}}\right)
$$

and we obtain a bound on the vertex defined at $\Lambda_{M}$ for the particular momenta at which the renormalization condition is set. Using Taylor's formula at $\Lambda=\Lambda_{M}$ we can verify ii) for $\Lambda=\Lambda_{M}$, and obtain a boundary condition on the vertex at $\Lambda_{M} ;\left\|V_{0,4}^{r, s, t}\left(\Lambda_{M}\right)\right\|_{\Lambda_{M}} \leq P \log \left(\frac{\Lambda_{M}}{\Lambda_{m}}\right)$. Using this 
boundary condition it is then straightforward to verify ii) and iii) for $V_{0,4}^{r, s, t}(\Lambda)$ in the same way as these bounds were verified for the irrelevant vertices. Thus, ii) and iii) are verified for the four-point vertex with $\Phi$ legs only.

Exactly the same argument works for $V_{2,2}^{r, s, t}(\Lambda)$ and for $\partial_{p}^{2} V_{0,2}^{r, s, t}(\Lambda)$. We may then verify iv) for $\left\|\partial_{p} V_{0,2}^{r, s, t}(\Lambda)\right\|_{\Lambda_{M}}$ by using the bounds on $\left\|\partial_{p}^{2} V_{0,2}^{r, s, t}(\Lambda)\right\|_{\Lambda_{M}}$ and Taylor's formula about zero momentum. It is then straightforward to verify iv) for $\left\|V_{0,2}^{r, s, t}(0)\right\|_{\Lambda_{M}}$ by using the boundary condition on the vertex at $\Lambda_{0}$ and Taylor's formula, obtaining $\left\|V_{0,2}^{r, s, t}(0)\right\|_{\Lambda_{M}} \leq \Lambda_{M}^{2} P \log \left(\frac{\Lambda_{M}}{\Lambda_{m}}\right)$. Using this boundary condition on the vertex we may verify iv) for all $\Lambda \in\left[0, \Lambda_{m}\right]$ by integrating the left-hand side of (2.3) from 0 to $\Lambda$ and using (2.13). Also, using the boundary condition from evaluating (vi) at $\Lambda_{m}$, we then verify iv) for all $\Lambda \in\left[\Lambda_{m}, \Lambda_{M}\right]$ by integrating (2.3) from $\Lambda_{m}$ up to $\Lambda$ and using (2.11). In this way we may verify ii), iii) and iv) for all the relevant vertices with $n>0$.

We can then prove iii) for the relevant vertices involving only $\phi$ fields. The method for doing this is essentially the same as in [1]. Once we use the bounds obtained from iii) in order to bound the term involving $\left\|V_{2 m, 2}^{r, s, t}(\Lambda)\right\|_{\Lambda, \Lambda_{M}}$, the right-hand side of (2.3) is consistent with iii), i.e we obtain the result in (2.15). Thus, it simply remains to verify i) for the relevant vertices, and ii) with $n=0$.

e) In order to prove ii) for the relevant vertices with $n=0$ we use the same method as used in part d) of lemma 1, i.e. we integrate the left-hand side of (2.3) defined at the momenta at which the renormalization conditions were set from $\Lambda \in\left[\Lambda_{m}, \Lambda_{M}\right]$ down to $\Lambda_{m}$, and use the derived boundary condition on the coupling constant at $\Lambda_{m}$ (obtained from iii) evaluated at $\Lambda_{m}$ for the particular momenta) to obtain a bound on the coupling constant defined at $\Lambda$. For example, we find that $\left|V_{4,0}^{r, s, t}\left(P_{i} ; \Lambda\right)\right| \leq P \log \left(\frac{\Lambda}{\Lambda_{m}}\right)+\frac{\Lambda}{\Lambda_{0}} P \log \left(\frac{\Lambda_{m}}{\Lambda_{0}}\right)$. We may then verify ii) by using these results along with Taylor's formula. We simply have to remember to work downwards in number of legs, and then in number of derivatives.

f) Finally, we may verify i) for all the relevant vertices in the same manner. We simply integrate the $\Lambda$-derivative of the coupling constants from $\Lambda$ down to $\Lambda_{M}$, use the derived boundary conditions on these couplings at $\Lambda_{M}$, along with the bound on the $\Lambda$-derivative, to provide a bound on the coupling constant defined at $\Lambda$, and use Taylor's formula to derive the bound on the norm of the vertex. Again, we must do this first for the four-point vertices, and then for the two-point vertices in decreasing number of derivatives (though for given number of legs it does not matter whether we deal with vertices with $\phi$ or $\Phi$ legs first). Once this is complete we have verified i)-iv) for all vertices.

We have thus demonstrated that lemma 8 is true for all $m$ and $n$ at orders $r, s$ and $t$ in the expansion coefficients. At next order in any of these coefficients the lemma is true at large enough $m$ and $n$, and again the proof by induction goes through and the lemma is true at this next order. But the lemma is trivially satisfied at zeroth order in all the expansion coefficients, and thus, by induction, lemma 8 is true for all $m, n, r, s$ and $t$. 
We have therefore been able to obtain a bound on all the vertices for all $\Lambda$ up to, and including, $\Lambda_{0}$. In particular, iii) and iv) evaluated at $\Lambda=0$ tell us that for $n>0$, for all $m$, $j$, except for $m=0, n=1$, and $j=0,1$,

$$
\left\|\partial_{p}^{j} \widetilde{G}_{2 m, 2 n}^{c}\left(\Lambda_{0}, \lambda_{i}\right)\right\|_{\Lambda_{m}, \Lambda_{M}} \leq \Lambda_{m}^{4-2 m-2 n-j} P \log \left(\frac{\Lambda_{M}}{\Lambda_{m}}\right)
$$

for $n=0$, for all $m, j$,

$$
\left\|\partial_{p}^{j} \widetilde{G}_{2 m, 0}^{c}\left(\Lambda_{0}, \lambda_{i}\right)\right\|_{\Lambda_{m}} \leq \Lambda_{m}^{4-2 m-j}
$$

and for the special case $m=0, n=1, j=0,1$, the heavy two-point vertex satisfies

$$
\left\|\partial_{p}^{j} \widetilde{G}_{0,2}^{c}\left(\Lambda_{0}, \lambda_{i}\right)\right\|_{\Lambda_{M}} \leq \Lambda_{M}^{2-j} P \log \left(\frac{\Lambda_{M}}{\Lambda_{m}}\right) .
$$

These bounds show that the amputated connected Green's functions have a maximum value which is independent of $\Lambda_{0}$, and thus that they remain finite in the limit $\Lambda_{0} \rightarrow \infty$.

\subsection{Convergence and Universality.}

By combining the methods used to prove the convergence and universality of Green's functions in the infinite cut-off limit in [1] (lemma 2 and lemma 3 respectively) with those described above to prove lemma 8 , it is now relatively straightforward to prove convergence and universality lemmas for the theory under consideration here. The proofs of these lemmas will thus be omitted.

The convergence lemma takes the form

Lemma 9:

For $n>0$, for all $m, j$, except for $m=0, n=1$, and $j=0,1$,

$$
\left\|\left(\Lambda_{0} \frac{\partial}{\partial \Lambda_{0}} \partial_{p}^{j} \widetilde{G}_{2 m, 2 n}^{c}\left(\Lambda_{0}, \lambda_{i}\right)\right)_{\lambda_{i}}\right\|_{\Lambda_{m}, \Lambda_{M}} \leq\left(\frac{\Lambda_{M}}{\Lambda_{0}}\right)^{2} \Lambda_{m}^{4-2 m-2 n-j} P \log \left(\frac{\Lambda_{M}}{\Lambda_{m}}\right) P \log \left(\frac{\Lambda_{0}}{\Lambda_{m}}\right)
$$

while for $n=0$, for all $m, j$,

$$
\left\|\left(\Lambda_{0} \frac{\partial}{\partial \Lambda_{0}} \partial_{p}^{j} \widetilde{G}_{2 m, 0}^{c}\left(\Lambda_{0}, \lambda_{i}\right)\right)_{\lambda_{i}}\right\|_{\Lambda_{m}} \leq\left(\frac{\Lambda_{m}}{\Lambda_{0}}\right)^{2} \Lambda_{m}^{4-2 m-j} P \log \left(\frac{\Lambda_{0}}{\Lambda_{m}}\right),
$$

and for the special case $m=0, n=1, j=0,1$,

$$
\left\|\left(\Lambda_{0} \frac{\partial}{\partial \Lambda_{0}} \partial_{p}^{j} \widetilde{G}_{0,2}^{c}\left(\Lambda_{0}, \lambda_{i}\right)\right)_{\lambda_{i}}\right\|_{\Lambda_{M}} \leq\left(\frac{\Lambda_{M}}{\Lambda_{0}}\right)^{2} \Lambda_{M}^{2-j} P \log \left(\frac{\Lambda_{M}}{\Lambda_{m}}\right) P \log \left(\frac{\Lambda_{0}}{\Lambda_{m}}\right) .
$$

Thus the connected amputated Green's functions, and hence the S-matrix elements, calculated using the effective Lagrangian at any scale between $\Lambda_{m}$ and $\Lambda_{M}$ have a well defined limit as $\Lambda_{0} \rightarrow$ $\infty$. Therefore our theory containing particles of masses $m$ and $M$ is perturbatively renormalizable in the conventional sense. 
To prove universality we consider, just as in lemma 3, a second theory with more general irrelevant boundary conditions at $\Lambda_{0}$ than those of the first, (1.4), namely

$$
\begin{aligned}
\bar{V}_{2 m, 2 n}^{r, s, t}\left(p,-p ; \Lambda_{0}\right)=\bar{\lambda}_{2 m, 2 n}^{r, s, t}\left(\Lambda_{0}\right) \Lambda_{0}^{2}+p^{2} \bar{\lambda}_{2 m, 2 n}^{r, s, t}\left(\Lambda_{0}\right)+\Lambda_{0}^{2} \eta_{2 m, 2 n}^{r, s, t}\left(p,-p ; \Lambda_{0}\right), & m+n=1, \\
\bar{V}_{2 m, 2 n}^{r, s, t}\left(p_{1}, p_{2}, p_{3}, p_{4} ; \Lambda_{0}\right)=\bar{\lambda}_{2 m, 2 n}^{r, s, t}\left(\Lambda_{0}\right)+\eta_{2 m, 2 n}^{r, s, t}\left(p_{1}, p_{2}, p_{3}, p_{4} ; \Lambda_{0}\right), & m+n=2, \\
\bar{V}_{2 m, 2 n}^{r, s, t}\left(p_{1}, \cdots, p_{2(m+n)} ; \Lambda_{0}\right)=\Lambda_{0}^{4-2(m+n)} \eta_{2 m, 2 n}^{r, s, t}\left(p_{1}, \cdots, p_{2(m+n)} ; \Lambda_{0}\right), & m+n>2 .
\end{aligned}
$$

The functions $\eta_{2 m, 2 n}^{r, s, t}\left(p_{i} ; \Lambda_{0}\right)$ satisfy the same general conditions as those in $\S 3.1$ of [1], namely they are real functions of the momenta $p_{i}$, regular when continued into the complex plane, are natural in the sense that

$$
\left\|\partial_{p}^{j} \eta_{2 m, 2 n}^{r, s, t}\left(\Lambda_{0}\right)\right\|_{\Lambda_{0}} \leq \Lambda_{0}^{-j} P \log \left(\frac{\Lambda_{0}}{\Lambda_{M}}\right)
$$

for $2(m+n)+j>4$, and vanish for $m+n>r+s+t+1$. By convention we also take $\eta_{2 m, 2 n}^{r, s, t}\left(\Lambda_{0}\right)=0$ if $m=0$ and $r>0$, or if $n=0$ and $s>0$, or if $m n=0$ and $r s>0$.

We can then show that if we write $\Delta \widetilde{G}_{2 m, 2 n}^{c}$ for the difference between the amputated connected Green's functions of the two theories,

Lemma 10:

For $n>0$, for all $m, j$, except for $m=0, n=1$, and $j=0,1$

$$
\left\|\partial_{p}^{j} \Delta \widetilde{G}_{2 m, 2 n}^{c}\right\|_{\Lambda_{m}, \Lambda_{M}} \leq\left(\frac{\Lambda_{M}}{\Lambda_{0}}\right)^{2} \Lambda_{m}^{4-2 m-2 n-j} P \log \left(\frac{\Lambda_{M}}{\Lambda_{m}}\right) P \log \left(\frac{\Lambda_{0}}{\Lambda_{m}}\right)
$$

while for $n=0$, for all $m, j$,

$$
\left\|\partial_{p}^{j} \Delta \widetilde{G}_{2 m, 0}^{c}\right\|_{\Lambda_{m}} \leq\left(\frac{\Lambda_{m}}{\Lambda_{0}}\right)^{2} \Lambda_{m}^{4-2 m-j} P \log \left(\frac{\Lambda_{0}}{\Lambda_{m}}\right)
$$

and for the special case $m=0, n=1, j=0,1$,

$$
\left\|\partial_{p}^{j} \Delta \widetilde{G}_{0,2}^{c}\right\|_{\Lambda_{M}} \leq\left(\frac{\Lambda_{M}}{\Lambda_{0}}\right)^{2} \Lambda_{M}^{2-j} P \log \left(\frac{\Lambda_{M}}{\Lambda_{m}}\right) P \log \left(\frac{\Lambda_{0}}{\Lambda_{m}}\right) .
$$

Thus all S-matrix elements are universal, in the sense that they are independent, up to power suppressed terms, of all the irrelevant parameters $\eta_{2 m, 2 n}^{r, s, t}\left(p_{i} ; \Lambda_{0}\right)$, provided that these are natural at $\Lambda_{0}$. The effective theory of the two particles is thus renormalizable in the precise sense explained in [1]. As expected from power counting, corrections to light particle processes are suppressed $\Lambda_{m}^{2} / \Lambda_{0}^{2}$ (up to logarithms), while those involving heavy particles are only suppressed by powers of $\Lambda_{M}^{2} / \Lambda_{0}^{2}$.

Both lemma 9 and lemma 10 may be systematically improved, after the fashion of $§ 3.2$ of [1]. We here defer discussion of systematic improvement until $\S 4$. 


\subsection{Weinberg Bounds.}

The bound (2.19) derived from lemma 8 is actually rather weak; by considering in more detail the number of heavy particle legs on a given vertex, rather than just splitting vertices into those with either some heavy particle legs or no heavy particle legs, it is possible to obtain much more stringent bounds on the Green's functions with heavy particle legs (we already have the best possible bound, namely (2.20), for Green's functions with light particle legs only). To do this we need to also incorporate the notation and arguments in ref.[4] in order to consider the number $\widetilde{e}$ of heavy particle exceptional momenta for a particular vertex (i.e. the total number of momenta within all irreducible sets (except for the largest) which have magnitudes between some minimum value $E$ and $\Lambda_{M}$ ). The resulting bounds, analogous to lemma 6 , are summarized in the following lemma:

\section{Lemma 11:}

i) For all $m>0, n>1$, and $0 \leq \widetilde{e} \leq 2 n-3$

$$
\left\|\partial_{p}^{j} \widetilde{G}_{2 m, 2 n}^{c}\left(\Lambda_{0}, \lambda_{i}\right)\right\|_{\Lambda_{m}, \Lambda_{M}}^{E, \widetilde{e}} \begin{cases}\leq \Lambda_{M}^{3+\widetilde{e}-2 n} \Lambda_{m}^{2-2 m-j} \bar{\Lambda}^{-1-\widetilde{e}} P \log \left(\frac{\Lambda_{M}}{\Lambda_{m}}\right) & \widetilde{e} \text { odd } \\ \leq \Lambda_{M}^{2+\widetilde{e}-2 n} \Lambda_{m}^{2-2 m-j} \bar{\Lambda}^{-\widetilde{e}} P \log \left(\frac{\Lambda_{M}}{\Lambda_{m}}\right) & \tilde{e} \text { even }\end{cases}
$$

where $\bar{\Lambda}=\max \left(E, \Lambda_{m}\right)$;

ii) for $m=0$, and $n>0$, except for $n=1, j=0,1$, then for $\widetilde{e}=0$

$$
\left\|\partial_{p}^{j} \widetilde{G}_{0,2 n}^{c}\left(\Lambda_{0}, \lambda_{i}\right)\right\|_{\Lambda_{m}, \Lambda_{M}} \leq \Lambda_{M}^{4-2 n-j}
$$

while for $1 \leq \widetilde{e} \leq 2 n-3$,

$$
\left\|\partial_{p}^{j} \widetilde{G}_{0,2 n}^{c}\left(\Lambda_{0}, \lambda_{i}\right)\right\|_{\Lambda_{m}, \Lambda_{M}}^{E, \widetilde{e}} \begin{cases}\leq \Lambda_{M}^{3+\widetilde{e}-2 n} \bar{\Lambda}^{1-\widetilde{e}-j} P \log \left(\frac{\Lambda_{M}}{\bar{\Lambda}}\right) & \widetilde{e} \text { odd } \\ \leq \Lambda_{M}^{2+\widetilde{e}-2 n} \bar{\Lambda}^{2-\widetilde{e}-j} P \log \left(\frac{\Lambda_{M}}{\bar{\Lambda}}\right) & \widetilde{e} \text { even }\end{cases}
$$

iii) for $m=0, n=1, j=0,1$,

$$
\left\|\partial_{p}^{j} \widetilde{G}_{0,2}^{c}\left(\Lambda_{0}, \lambda_{i}\right)\right\|_{\Lambda_{M}} \leq \Lambda_{M}^{2-j}
$$

iv) for all $m>0, n=1$,

$$
\left\|\partial_{p}^{j} \widetilde{G}_{2 m, 2}^{c}\left(\Lambda_{0}, \lambda_{i}\right)\right\|_{\Lambda_{m}, \Lambda_{M}} \leq \Lambda_{m}^{4-2 m-j} P \log \left(\frac{\Lambda_{M}}{\Lambda_{m}}\right)
$$

Here $\widetilde{e}$ denotes the maximum number of exceptional momenta for the external legs corresponding to heavy particles; all the external momenta for light particle legs can be individually exceptional as low as zero. All derivatives are assumed to act on light particle legs, or else heavy particle legs carrying exceptional momenta (if this is not the case they would produce inverse powers of $\Lambda_{M}$ rather than $\left.\bar{\Lambda}\right)$. The vertices with $m>0$ and $n=1$ are a special case because the large number of light particle legs mean that the total number of possible exceptional momenta already exceeds $2 m+2 n-3$. It is convenient to have these best possible bounds, since then the bounds are 
comparable to the renormalization conditions on the vertices involving heavy particles. ${ }^{4}$ However as lemma 8 is sufficient to prove the decoupling theorem, we leave the proof of lemma 11 (a straightforward, but rather lengthy, combination of the methods of this paper and [4]) to the more enthusiastic reader.

\section{The Decoupling Theorem.}

In the context of the effective scalar field theories discussed so far, the decoupling theorem consists of the following statement: if we calculate connected amputated Green's functions involving only light particles at scales $\sim \Lambda_{m}$, using an effective theory containing only $\phi$ fields (as defined in [1]), with naturalness scale $\Lambda_{M}$, then these Green's functions will be the same as those calculated using the full theory (as defined in $\S 1$ above), up to corrections suppressed by powers of $\left(\Lambda_{m} / \Lambda_{M}\right)$.

When viewed in this way, decoupling is nothing but a generalization of universality: the light particle theory with regular boundary conditions on its irrelevant vertices is approximately equivalent at low energies to one in which these vertices have heavy particle poles and cuts. Indeed the method we will use to prove the decoupling theorem is very similar to that used to prove universality in $\S 3.1$ of [1].

In this section we state and prove a lemma which contains the decoupling theorem in its most simple form. Then in the next section we will generalize this result. We thus introduce a theory containing only $\phi$ fields, and in fact defined precisely as in $\S 2.1$ of [1], with zero boundary conditions on the irrelevant couplings at $\Lambda_{M}$, and boundary conditions on the relevant couplings which are the same as (1.5) for the vertices containing only $\phi$ fields. We denote the vertices of this new theory by $\widehat{V}_{2 m}^{r, s, t}\left(p_{1} \ldots . . p_{2 m} ; \Lambda\right)$, and consider the difference between this theory and the one containing both $\phi$ and $\Phi$ fields by introducing the quantity

$$
D_{2 m}^{r, s, t}(\Lambda) \equiv V_{2 m, 0}^{r, s, t}(\Lambda)-\widehat{V}_{2 m}^{r, s, t}(\Lambda)
$$

which gives us a measure of the difference between the two theories.

Subtracting the flow equation for the $\widehat{V}_{2 m}^{r, s, t}(\Lambda)$ away from that for the $V_{2 m, 0}^{r, s, t}(\Lambda)$ and taking norms, we easily obtain, for $\Lambda \in\left[0, \Lambda_{M}\right]$,

$$
\begin{gathered}
\left\|\frac{\partial}{\partial \Lambda}\left(\partial_{p}^{j} D_{2 m}^{r, s, t}(\Lambda)\right)\right\|_{\Lambda^{\prime}} \leq \Lambda\left\|\partial_{p}^{j} D_{2 m+2}^{r, s, t}(\Lambda)\right\|_{\Lambda^{\prime}} \\
+\sum_{l=1}^{m} \sum_{r^{\prime}=1}^{r-1} \sum_{s^{\prime}=1}^{s-1} \sum_{t^{\prime}=1}^{t-1} \sum_{j_{i} ; j_{1}+j_{2}+j_{3}=j} \Lambda^{-3-j_{1}}\left[\left\|\partial_{p}^{j_{2}} D_{2 l}^{r^{\prime}, s^{\prime}, t^{\prime}}(\Lambda) \Lambda\right\|\left\|_{p}^{j} V_{2 m, 2}^{r, s, t}(\Lambda)\right\|_{\Lambda^{\prime}, \Lambda_{M}}\right. \\
\left.+\left\|\partial_{p}^{j_{2}} \widehat{V}_{2 l}^{r^{\prime}, s^{\prime}, t^{\prime}}(\Lambda)\right\|_{\Lambda^{\prime}}\right] \cdot\left\|\partial_{p}^{j_{3}} D_{2 m+2-2 l}^{r-r^{\prime}, s-s^{\prime}, t-t^{\prime}}(\Lambda)\right\|_{\Lambda^{\prime}}
\end{gathered}
$$

4 Though in practice the on-shell renormalization conditions for vertices with heavy particle legs may be larger than the corresponding Euclidean space bounds because of the proximity of the poles in the Green's functions to branch points due to light particle bremmstrahlung. 
where again $\Lambda^{\prime}=\max \left(\Lambda, \Lambda_{m}\right)$ just as in (2.3).

If we consider $\Lambda \in\left[\Lambda_{m}, \Lambda_{M}\right]$, we have the equality

$$
\partial_{p}^{j} D_{2 m}^{r, s, t}(\Lambda)-\partial_{p}^{j} D_{2 m}^{r, s, t}\left(\Lambda_{M}\right)=\int_{\Lambda}^{\Lambda_{M}} d \Lambda^{\prime} \frac{\partial}{\partial \Lambda^{\prime}}\left(\partial_{p}^{j} D_{2 m}^{r, s, t}\left(\Lambda^{\prime}\right)\right)
$$

which in the same way as previous equalities is easily turned into the inequality

$$
\left\|\partial_{p}^{j} D_{2 m}^{r, s, t}(\Lambda)\right\|_{\Lambda} \leq\left\|\partial_{p}^{j} D_{2 m}^{r, s, t}\left(\Lambda_{M}\right)\right\|_{\Lambda_{M}}+\int_{\Lambda}^{\Lambda_{M}} d \Lambda^{\prime}\left\|\frac{\partial}{\partial \Lambda^{\prime}}\left(\partial_{p}^{j} D_{2 m}^{r, s, t}\left(\Lambda^{\prime}\right)\right)\right\|_{\Lambda^{\prime}} .
$$

This equation is useful for bounding the difference between the irrelevant vertices for our two theories for $\Lambda \in\left[\Lambda_{m}, \Lambda_{M}\right]$. For $\Lambda \in\left[0, \Lambda_{m}\right]$ we can derive similarly

$$
\left\|\partial_{p}^{j} D_{2 m}^{r, s, t}(\Lambda)\right\|_{\Lambda_{m}} \leq\left\|\partial_{p}^{j} D_{2 m}^{r, s, t}\left(\Lambda_{m}\right)\right\|_{\Lambda_{m}}+\int_{\Lambda}^{\Lambda_{m}} d \Lambda^{\prime}\left\|\frac{\partial}{\partial \Lambda^{\prime}}\left(\partial_{p}^{j} D_{2 m}^{r, s, t}\left(\Lambda^{\prime}\right)\right)\right\|_{\Lambda_{m}} .
$$

To obtain equations which are useful for finding bounds on the difference between the relevant coupling constants in the range $\Lambda \in\left[\Lambda_{m}, \Lambda_{M}\right]$ it is necessary to integrate with respect to $\Lambda$ down to $\Lambda=\Lambda_{m}$, put the momenta equal to those at which the renormalization conditions on the relevant coupling constants are set, and take bounds to obtain

$$
\left.\left|\partial_{p}^{j} D_{2 m}^{r, s, t}(\Lambda)\right|_{p_{i}=P_{i}}|\leq| \partial_{p}^{j} D_{2 m}^{r, s, t}\left(\Lambda_{m}\right)\right|_{p_{i}=P_{i}} \mid+\int_{\Lambda_{m}}^{\Lambda} d \Lambda^{\prime}\left\|\frac{\partial}{\partial \Lambda^{\prime}} \partial_{p}^{j} D_{2 m}^{r, s, t}\left(\Lambda^{\prime}\right)\right\|_{\Lambda^{\prime}} .
$$

For an equation useful for finding bounds on the difference between the relevant vertices for our two theories for $\Lambda \in\left[0, \Lambda_{m}\right]$, we must integrate from $\Lambda$ down to 0 and take bounds with respect to $\Lambda_{m}$ to obtain

$$
\left\|\partial_{p}^{j} D_{2 m}^{r, s, t}(\Lambda)\right\|_{\Lambda_{m}} \leq\left\|\partial_{p}^{j} D_{2 m}^{r, s, t}\left(\Lambda_{m}\right)\right\|_{\Lambda_{m}}+\int_{0}^{\Lambda} d \Lambda^{\prime}\left\|\frac{\partial}{\partial \Lambda^{\prime}} \partial_{p}^{j} D_{2 m}^{r, s, t}\left(\Lambda^{\prime}\right)\right\|_{\Lambda_{m}} .
$$

Equations (3.2) -(3.7), together with the values of the vertices at $\Lambda_{M}$ and the bounds on the vertices in both theories, which are already known from lemmas 1 and 5 (since the theory containing just the $\phi$ fields is the same as that in [1] with $\Lambda_{m}=\Lambda_{R}, \Lambda_{M}$ instead of $\Lambda_{0}$ ), will now be shown to lead to a bound on the difference between the vertices, and thus the Green's functions, in the two theories. These bounds are summarized in

Lemma 12:

i) For all $\Lambda \in\left[\Lambda_{m}, \Lambda_{M}\right]$,

$$
\left\|\partial_{p}^{j} D_{2 m}^{r, s, t}(\Lambda)\right\| \leq \frac{\Lambda}{\Lambda_{M}} \Lambda^{4-2 m-j} P \log \left(\frac{\Lambda_{M}}{\Lambda_{m}}\right)
$$

ii) For all $\Lambda \in\left[0, \Lambda_{m}\right]$,

$$
\left\|\partial_{p}^{j} D_{2 m}^{r, s, t}(\Lambda)\right\|_{\Lambda_{m}} \leq \frac{\Lambda_{m}}{\Lambda_{M}} \Lambda_{m}^{4-2 m-j} P \log \left(\frac{\Lambda_{M}}{\Lambda_{m}}\right) .
$$


Once again, the proof follows the same induction scheme as that used to prove lemma 1; we assume that the lemma is true at order $r-1, s-1, t-1$ in $g_{m}, g_{M}$ and $g_{m M}$, and vertices with more than $2 m+2$ legs at next order in the coupling constants, and then show that it remains true for vertices with $2 m$ legs at order $r, s, t$. Each induction step then follows the same four steps a)-d) as lemma 1.

a) For the irrelevant vertices with $\Lambda \in\left[\Lambda_{m}, \Lambda_{M}\right]$ we simply have to use (3.4) and the fact that the values of the vertices in each theory at $\Lambda_{M}$ are consistent with lemma 12 , and therefore so is their difference, as we see by substituting $\Lambda=\Lambda_{M}$ into lemma 8ii), and i) is immediately seen to be true at this order in the expansion parameters for vertices with $2 m$ legs. The second term in (3.2) causes no problem since, using (2.5) we see that it is bounded by $K^{1 / 2}\left(\frac{M^{2}}{\Lambda^{2}}\right) \Lambda^{3-2 m-j} P \log \left(\frac{\Lambda_{M}}{\Lambda_{m}}\right)$, which is less than or equal to the bound $\Lambda^{3-2 m-j}\left(\frac{\Lambda}{\Lambda_{M}}\right) P \log \left(\frac{\Lambda_{M}}{\Lambda_{m}}\right)$ required of all terms on the right-hand side of (3.2) in order for the induction argument used to prove i) to go through.

b) The proof of ii) for the irrelevant vertices is much the same. We use the derived boundary conditions obtained by evaluating i) at $\Lambda_{m}$ along with (3.5), and ii) is clearly verified. As in a) the second term in (3.2) is no obstruction since, using (2.6) and the fact that the vertex has an associated factor of $K^{1 / 2}\left(\frac{\Lambda_{M}^{2}}{\Lambda^{2}}\right)$, we see that it is bounded by $\Lambda_{m}^{3-2 m-j}\left(\frac{\Lambda_{m}}{\Lambda_{M}}\right) P \log \left(\frac{\Lambda_{M}}{\Lambda_{m}}\right)$.

c) The difference between the relevant coupling constants in the two theories is zero by definition. Using this result along with Taylor's formula and the bounds already obtained for the irrelevant vertices we can prove ii) for each of the relevant vertices, by working down in number of legs and number of derivatives. In order to prove ii) for the relevant vertices for all $\Lambda \in\left[0, \Lambda_{m}\right]$ we use the derived boundary conditions at $\Lambda=0$ along with (3.7), again working downwards in number of legs and number of derivatives. The potentially troublesome second term in (3.2) is dealt with in the same way as in b).

d) From ii) there are now good bounds on the difference between the relevant coupling constants at $\Lambda_{m}$. Feeding these into (3.6) it is simply a repetition of previous exercises to bound the relevant couplings and to prove the lemma for the relevant vertices, using Taylor's formula and the same methods used for the relevant vertices in $\S$ 's 3.1 and 3.2. Once this is done, then as explained in these previous sections, the proof by induction is complete, and lemma 12 is true for all $r, s, t$ and $m$.

In particular, setting $\Lambda=0$, lemma 12 yields

$$
\left\|\partial_{p}^{j}\left(\widetilde{G}_{2 m, 0}^{c}\left(\Lambda_{0}, \lambda_{i}\right)-\widehat{G}_{2 m}^{c}\left(\Lambda_{M}, \lambda_{i}\right)\right)\right\|_{\Lambda_{m}} \leq \frac{\Lambda_{m}}{\Lambda_{M}} \Lambda_{m}^{4-2 m-j} P \log \left(\frac{\Lambda_{M}}{\Lambda_{m}}\right)
$$

where the amputated connected Green's functions for the theory with both light and heavy particles $\widetilde{G}_{2 m, 0}^{c}$ depend on $\lambda_{i}$, the seven coupling constants corresponding to the relevant vertices with either (or both) light and heavy particle legs, while the amputated connected Green's functions for the light particle theory, $\widehat{G}_{2 m}^{c}$, depend only on the three coupling constants corresponding to the relevant vertices with light particle legs only. Thus, we see that if we simply delete the heavy particle fields $\Phi$ from the original theory, while keeping the renormalization conditions on the 
relevant couplings at $\Lambda=0$ fixed, we change the amputated connected Green's functions including only $\phi$ fields at external momenta of order $\Lambda_{m}$ by terms of order $\Lambda_{m} / \Lambda_{M}$. This is particularly transparent if we adopt the convention described following (1.5). In this case the vertices in the theory containing only the light particle have an expansion in $g_{m}$ only, and we see from (3.9) that all terms in the low-energy light particle vertices in the full theory at greater than zeroth order in either $g_{M}$ or $g_{m M}$ are suppressed by $\Lambda_{m} / \Lambda_{M}$.

Thus, if we were to take $M / m \rightarrow \infty$ lemma 12 would therefore amount to a proof of the conventional decoupling theorem[5]. Combining lemma 12 with the universality of both two theories (lemmas 3 and 10) also gives us trivially a proof of decoupling in effective theories. More subtly, combining it with the proof of infrared finiteness of the light particle theory in [4] (lemma 5) gives us a proof of the decoupling theorem when the light particles are massless; this is very difficult using more conventional techniques[6].

\section{Systematic Improvement.}

Since in any realistic scenario the mass of the heavy particle is not infinite but finite, it would be useful to improve our decoupling theorem in much the same way that we improved the renormalizability of the effective theory in $\S 3.2$ of [1]. There we were able to show that one can decrease the dependence of low energy Green's functions on the irrelevant couplings at the naturalness scale $\Lambda_{0}$ by specifying more and more low energy renormalization conditions, and thus determining the coupling constants corresponding to higher and higher dimension operators. Here we might expect that it would be possible to decrease the dependence of the Green's functions for the low mass particle on the details of the theory of large mass particle $M$ by specifying more and more renormalization conditions on the theory containing only the light particle. It is easy to verify that this is indeed the case.

First we consider the theory containing both particles. Consider setting renormalization conditions on all irrelevant vertices up to a given canonical dimension $\widetilde{D}$. As with (1.5), these must be set with light particle legs having momenta of order $\Lambda_{m}$, and heavy particle legs with momenta of order $\Lambda_{M}$. We can apply the same arguments as used in [1] to show that the bounds in lemma 8 are still satisfied, while those in lemmas 9 and 10 are improved: (2.22), (2.24), (2.27), and (2.29)

all acquire an extra factor of $\left(\frac{\Lambda_{M}}{\Lambda_{0}}\right)^{\widetilde{D}-4}$ on the right hand side, while (2.23) and (2.28) acquire an extra factor of $\left(\frac{\Lambda_{m}}{\Lambda_{0}}\right)^{\widetilde{D}-4}$.

To improve the decoupling theorem, we repeat the argument in the previous section, with the physically relevant vertices in the light particle theory (all vertices with dimension not exceeding $D$, where for the moment we let $D=\widetilde{D}$ ) having identical low energy renormalization conditions to the vertices involving light particles alone in the theory containing both particle. For simplicity the remaining irrelevant vertices (with dimension greater than $D$ ) are set to zero at $\Lambda_{M}$ in the light particle theory, as are the undetermined irrelevant vertices at $\Lambda_{0}$ in the full theory. Lemma 12 is then superseded by the following improved bounds: 
Lemma 13:

(i) For all $\Lambda \in\left[\Lambda_{m}, \Lambda_{M}\right]$,

$$
\left\|\partial_{p}^{j} D_{2 m}^{r, s, t}(\Lambda)\right\| \leq\left(\frac{\Lambda}{\Lambda_{M}}\right)^{D-2} \Lambda^{4-2 m-j} P \log \left(\frac{\Lambda_{M}}{\Lambda_{m}}\right) .
$$

(ii) For all $\Lambda \in\left[0, \Lambda_{m}\right]$,

$$
\left\|\partial_{p}^{j} D_{2 m}^{r, s, t}(\Lambda)\right\|_{\Lambda_{m}} \leq\left(\frac{\Lambda_{m}}{\Lambda_{M}}\right)^{D-2} \Lambda_{m}^{4-2 m-j} P \log \left(\frac{\Lambda_{M}}{\Lambda_{m}}\right) .
$$

In order to prove this lemma we may use exactly the same induction argument as that for lemma 12. Since both sets of boundary conditions on $\partial_{p}^{j} D_{2 m}^{r, s, t}(\Lambda)$ are consistent with the lemma, and the flow equation (3.2) is also consistent with it (partly due to the exponential in the second term on the right falling of more quickly than any finite power), the proof goes through just as before, and we see no reason to write it out in detail. Just as for lemma 12 the assumptions on the irrelevant vertices may be relaxed using the (improved) universality lemmas 4 and 10.

Setting $\Lambda=0$ in (4.2), we see immediately that (3.10) now becomes

$$
\left\|\partial_{p}^{j}\left(\widetilde{G}_{2 m, 0}^{c}\left(\Lambda_{0}, \lambda_{i}\right)-\widehat{G}_{2 m}^{c}\left(\Lambda_{M}, \lambda_{i}\right)\right)\right\|_{\Lambda_{m}} \leq\left(\frac{\Lambda_{m}}{\Lambda_{M}}\right)^{D-2} \Lambda_{m}^{4-2 m-j} P \log \left(\frac{\Lambda_{M}}{\Lambda_{m}}\right)
$$

the amputated connected Green's functions for the two theories only differ by terms of order $\left(\frac{\Lambda_{m}}{\Lambda_{M}}\right)^{D-2}$ (up to logarithms) when we consider external momenta with magnitudes of order $\Lambda_{m}$. So setting renormalization conditions on light particle couplings down to dimension $4-D$ means that we can calculate amputated connected Green's functions (and thus S-matrix elements) for scatterings of light particles with energies of order $\Lambda_{m}$ simply by using a theory containing just the light particle field with precision of order $\left(\frac{\Lambda_{m}}{\Lambda_{M}}\right)^{D-2}$. Thus, as far as the bounding arguments are concerned, we have shown that the naturalness scale $\Lambda_{0}$ in [1] could equally be the mass of another particle: all the results proven in $\S 2$ and $\S 3$ of this paper sections being also true in this case, as we might naively have expected.

However the physically relevant renormalization conditions for the light particles are now dictated, up to small corrections, by matching to the theory with the heavy particle: more precisely in the phenomenologically 'neutral' effective theory discussed in [1], if all renormalization conditions corresponding to vertices of dimension $D$ have been fixed, those of dimension $D+2$ may be specified within a freedom of order $\left(\frac{\Lambda_{m}}{\Lambda_{M}}\right)^{D-2}$ (as explained in $\S 3.3$ of [1]); matching to the massive theory means that this freedom is only of order $\left(\frac{\Lambda_{m}}{\Lambda_{0}}\right)^{D-2}$. This means, in fact, that we have the freedom to choose $D$ to be greater than $\widetilde{D}$, as long as it satisfies the requirement that $\left(\frac{\Lambda_{m}}{\Lambda_{0}}\right)^{\widetilde{D}-2} \leq\left(\frac{\Lambda_{m}}{\Lambda_{M}}\right)^{D-2}$. In this case we set the renormalization conditions for the light particle theory to be identical to those for the light particle vertices in the full theory for dimension up to $\widetilde{D}$ and equal to the values obtained by calculating using the full theory for those vertices of higher dimension. (If the renormalization conditions are set by matching to experiment this will be true automatically, up 
to corrections of order $\left(\frac{\Lambda_{m}}{\Lambda_{0}}\right)^{\widetilde{D}-2}$.) The proof of lemma 13 then goes through with no obstruction and the conclusions expressed in (4.3) and the following paragraph still hold.

In particular, in the local limit $\frac{\Lambda_{m}}{\Lambda_{0}} \rightarrow 0$, with $\frac{\Lambda_{m}}{\Lambda_{M}}$ held fixed, simply choosing $\widetilde{D}=4$ the matching conditions fix the renormalization conditions of the effective theory of the light particle alone precisely, and we may choose any $D \geq 4$; this generalizes the result of [7] to all $D>6$ with, it seems to us, remarkably little effort. Conversely, if $\Lambda_{0}$ is not very much larger than $\Lambda_{M}$, we must choose $D$ to be much the same as $\widetilde{D}$, and relatively little is gained in the precision of the light particle theory by matching it to the heavy particle one. Of course, we expect that in all realistic theories we will be somewhere between these two extremes.

Finally we consider stability. We can use the technique described in $\S 5$ of [1] to construct a large class of nonperturbatively stable theories containing only the light particle (with two scales $\Lambda_{m} \ll \Lambda_{M}$ ), and also another large class containing both the light particle and a stable heavy particle $^{5}$ (now with three scales $\Lambda_{m} \ll \Lambda_{M} \ll \Lambda_{0}$ ), each with the same renormalization conditions on physically relevant couplings. It should be clear that both these theories will have S-matrices which, at least in perturbation theory, are both unitary and causal. Furthermore the S-matrix of the latter is unitary not only on the full space of light and heavy particle external states of arbitrary energy, but also on the subspace of light particle states with energies less than $2 M$, since from the cutting relation the heavy particles can only contribute to the imaginary parts of light particle amplitudes above threshold. There is no guarantee however that the light particle theories on this subspace, when analytically continued to describe light particle processes of arbitrarily high energies, will remain stable non-perturbatively. However it is not difficult to see from (4.3) that there always exists a stable light particle theory whose $\mathrm{S}$-matrix is arbitrarily close to that of the full theory when restricted to this subspace.

We may now consider a wider class of scalar field theories. In particular, if we were to relax the $Z_{2}$ symmetry of the heavy particle interactions, they would be free to decay into the light particles. We can only really do this in the effective theory, since as explained in $\S 6$ of [1] if the vacuum is to be stable then the couplings $g_{M}^{\prime}$ and $g_{m M}^{\prime}$ for vertices involving an odd number of heavy particles are bounded above (by $\left(M / \Lambda_{0}\right) g_{M}^{1 / 2}$ and $\left(M / \Lambda_{0}\right) g_{m M}^{1 / 2}$ respectively); in the local limit the $Z_{2}$ symmetry is thus restored. The unitarity and causality of theories in which a heavy particle is allowed to decay into lighter particles was discussed long ago by Veltman[9]; the issue is a subtle one since in principle only the light particles of such a theory may be considered as external states, the heavy particle pole being no longer on the physical sheet (though it approaches it in the limit $g_{m M} \rightarrow 0$ ). However, it can be shown that there exists a unitary and causal S-matrix for the scattering of the light particles alone. This S-matrix restricted to the subspace of light particle states with energies less than $M$ is manifestly unitary, and using the methods of [1] we may clearly construct an effective light particle theory whose S-matrix tends arbitrarily close to that of the full theory on this subspace, and the improved decoupling theorem still holds.

5 A single heavy particle cannot decay into light ones because of the global $Z_{2}$ symmetry imposed on the heavy particle field. 
The question of stability is a little more complicated for such theories. Veltman showed [9] that the light field equations of motion obtained by integrating out the unstable heavy particle give a stable vacuum for the light field. However, the manifestly stable light particle theory obtained from adiabatic quantization [1] may not be able to reproduce the light particle theory obtained by formally integrating out the heavy field at $\Lambda_{M}$, and then expanding all nonanalytic terms in powers of momenta. This is because the 'higher derivative' terms generated via adiabatic quantization come about purely from the radiative part of the renormalization group flow, while when the heavy field is integrated out such operators are generated from tree diagrams. Thus the 'higher derivative' operators in the latter may be larger than those we can feasibly produce in the former. This is not really an important restriction for the present theory because the coupling between the light and heavy particles is necessarily weak compared to the couplings in the light particle theory due to the stability condition. However, in certain regions of coupling space, and for theories with unstable particles in which there is no reason for the coupling leading to the heavy particle decay to be relatively small (as for example in electroweak theory) normal adiabatic quantization may not be sufficient to produce a suitable effective field theory. In these cases it is however possible to obtain a manifestly stable primordial action by first writing it in terms of both the heavy and light field and then integrating out the unstable heavy particle. Expanding all nonanalytic terms in powers of momenta, and matching their Taylor series (which will all have radiuses of convergence of at least $M$ ) to analytic vertex functions, we may then use adiabatic quantization to produce a manifestly unitary and causal effective theory. It is not difficult to see that at scales below $\Lambda_{M}$ this effective theory will be identical (order by order in powers of momenta) to the manifestly stable theory obtained by adiabatically quantizing the full theory and then decoupling the heavy fields at $\Lambda_{M}$.

We can also consider a theory in which not only the $Z_{2}$ symmetry of the heavy particle interactions is broken, but in which the $Z_{2}$ symmetry of the light particles is also broken at scales of order $\Lambda_{M}$ by its interactions with the heavy particle. Thus while the physically relevant renormalization conditions on Green's functions involving only light particles are still set at momenta of order $\Lambda_{m}$, they will no longer be $Z_{2}$ symmetric, since amplitudes with an odd number of light particle legs may be induced by corrections involving virtual heavy particles. It is not difficult to see that the result (4.3), when generalized to cover this softly broken case, now implies that the amputated connected Green's functions of the light particle theory with an odd number of legs $2 m+1$ must

be suppressed relative to those with $2 m$ legs by a factor of $\left(\frac{\Lambda_{m}}{\Lambda_{M}}\right)$. This result is a simple paradigm for the breaking of parity by the weak interaction.

\section{The Local Limit.}

When working with an effective quantum field theory it is usually simplest both conceptually and technically to keep the regularization scale $\Lambda$ below the naturalness scale $\Lambda_{0}$; indeed when constructing stable theories as described in $\S 5.2$ the theory was only formally quantized for $\Lambda \lesssim \Lambda_{0}$. This was particularly obvious in our discussion of decoupling in the previous section, where the 
naturalness scale $\Lambda_{M}$ of the light particle theory was identified with the mass of some new heavy particle. When formulated in this way, we are always free to choose the number of physically relevant couplings we wish to work with, quite independently of technical details such as the order at which we truncate our perturbation theory.

Of course it is not necessary to follow this approach; as Green's functions are by construction independent of $\Lambda$ we may use the full renormalization group equations to take $\Lambda / \Lambda_{0}$ to infinity with impunity. Furthermore as explained in [1] all physical quantities are independent of the from of the regulating function $K_{\Lambda}$, so in this limit it might seem reasonable to introduce an alternative regularization procedure, for example dimensional regularization. The effective theory would then closely resemble a local quantum field theory, and indeed this is the approach which is most frequently to be found in the original literature[8,10]. We feel that this approach obscures the relationship between physics at different scales and in particular more and more counterterms must be introduced as we work to higher and higher loop order, simply in order to render the theory finite (thus destroying some of the properties of conventional local field theory, such as manifest stability). Indeed none of the conjectures formulated in the framework of local effective theories have, to our knowledge, been rigorously proven.

We are perhaps best able to see how our approach relates to this 'conventional' approach if we consider it in the context of decoupling. In $\S 2$ we can consider the theory containing the two particles in the local limit $\Lambda_{0} / \Lambda_{m} \rightarrow \infty$; this does not change $\S 3$ at all if we consider the effective theory containing only the light particle to have naturalness scale $\Lambda_{M}$. More relevantly, we could instead set the boundary conditions on the irrelevant couplings of the light particle theory at $\Lambda_{0}$, and thus also take this theory in the local limit; the bounds lemma 1 will be unchanged because the low energy renormalization conditions on the relevant couplings are independent of $\Lambda_{0}$, and the proof of decoupling, lemma 11, goes ahead just as it did in $\S 3$.

The case for the improved decoupling theorem presented in $\S 8.3$ is not so simple. In order to match the physical renormalization conditions on the vertices with dimension $D>4$ in the light particle theory to those of the theory containing the heavy particle, they must be such as to be natural when evolved back to $\Lambda_{M}$. This means that they cannot remain natural if we evolve them above $\Lambda_{M}$ to $\Lambda_{0}$, but rather will begin to diverge as powers of $\Lambda_{0} / \Lambda_{M}$ (up to logarithms). In the local limit, we will thus find a theory with infinite counterterms which are just such as to guarantee that the renormalized insertions in the light particle theory reproduce the effects of the heavy particle. The light particle theory is then an effective theory in the conventional (local) formulation.

Unfortunately, the combination of scales (or scales and factors of $\epsilon^{-1}$ if one were to use dimensional regularization) now makes the scale dependence of the physics of the effective theory rather more difficult to disentangle. In particular, it is in general necessary to work with the full infinite set of counterterms, even though after renormalization operators with dimension greater than $D$ are discarded; even if the renormalized couplings corresponding to these operators were set to zero, the form of the flow equations means that they will be nonzero when evolved back to $\Lambda_{0}$, and infinite in the local limit. 
Consider, for example, computations in the loop expansion. The form of the flow equations shows us that to create the natural effective Lagrangian at $\Lambda_{M}$ from that at $\Lambda_{0}$ we can only decrease the number of legs of a vertex by forming loop diagrams, and each loop causes a maximum reduction of two legs. Therefore, a vertex with $2 m+2 n$ legs at $\Lambda_{0}$ will make a contribution to a vertex at $\Lambda_{M}$ at a power of $\hbar$ which is at least $n$ higher. So even if we only wish to work to an accuracy of order $\left(\frac{\Lambda_{m}}{\Lambda_{M}}\right)^{D-2}$, and therefore at retain renormalized vertices with at most $D$ legs, if we work at order $l$ in the loop expansion it will be necessary to include vertices at $\Lambda_{0}$ with $2 l+4$ legs, just to render the renormalized vertices finite. At arbitrarily high order, we thus need an arbitrarily large number of counterterms. Outside perturbation theory the number of counterterms would in general be infinite.

Of course, there may well be situations where for practical reasons the local formulation is advantageous. For example when making perturbative computations in theories with local or nonlinearly realized symmetries (as in [10]) dimensional regularization is convenient because it manifestly preserves such symmetries; in the flow equations they seem to be necessarily broken by the introduction of the regularization function. ${ }^{6}$ Similarly, nonperturbative computations in an effective theory could perhaps be performed on the lattice if the lattice spacing $a \ll \Lambda_{0}^{-1}$ (though there would in general be problems with spurious instabilities on the scale of the lattice spacing if the finite difference equations were truncated at finite order). But formal issues, which in general benefit from a clear separation of scales are, we feel, most readily formulated and proven within the quasi-local formulation of effective field theory as presented in [1].

\section{Predictivity.}

To summarize, we have shown that a theory of two scalar particles with $Z_{2} \times Z_{2}$ symmetry and well separated mass scales $\Lambda_{m} \ll \Lambda_{M} \ll \Lambda_{0}$ may be approximated arbitrarily closely at scales below $\Lambda_{M}$ by an effective theory containing the light particle alone. This decoupling theorem, which we proved to all orders in perturbation theory, may be readily extended to any theory of scalar and/or spin half particles, with global symmetries, since as explained in $\S 6$ of [1] no new ideas are then necessary. All that is required is that the particles are arranged in a hierarchy of mass scales $\Lambda_{m} \ll \Lambda_{M_{1}} \ll \Lambda_{M_{2}} \ll \cdots \ll \Lambda_{0}$; each scale may then be decoupled sequentially. The decoupling theorem will also remain valid for effective field theories set in space-times with dimensions other than four, such as Kaluza-Klein theories. Theories with spontaneously broken symmetries, nonlinearly realized symmetries, or local symmetries are more difficult to handle, however, and indeed for them the decoupling theorem may sometimes break down.

We consider finally the opposite problem of attempting to predict the existence of new particles at higher mass scales than current experiments. We showed in [1] that we can describe the physics of processes below a certain energy scale $\Lambda_{M}$, using an effective field theory containing only those fields which correspond to particles with masses $m$ below this scale. As we increase the energy at

\footnotetext{
6 Though clearly some complicated remnant of the symmetry must remain.
} 
which the physics is probed, we need more parameters to maintain the same precision. If we were to make no assumptions about the nature of the theory at $\Lambda_{M}$, all of these new parameters would be constrained only by naturalness. However, if there were a new particle at $\Lambda_{M}$, the new parameters would be more tightly constrained by the requirement that at $\Lambda_{M}$ the light particle theory matched to the new theory containing the extra particle. The higher the new naturalness scale, $\Lambda_{0}$, the tighter these constraints would be, and the easier it would be to guess the existence of the heavy particle from the accurate examination of light particle processes below its threshold; if $\Lambda_{0}$ were too low, it would be difficult to disentangle the effects at low energy of the heavy particle from those of the physics at $\Lambda_{0}$. Of course the new couplings will be easier to measure accurately if they break some global symmetry (as for example the $Z_{2}$ symmetry considered above); the observation of parity violating weak interactions, through both weak and neutral current interactions, was seen as good evidence for the existence of intermediate vector bosons, and likewise new insights are expected from the study of CP violation (although here there is as yet no compelling candidate for an underlying theory).

If the new heavy particle really existed, then as we reached the threshold for heavy particle production, our effective theory containing just the light particle would necessarily breakdown, since by construction it does not contain the threshold singularity. Although by using enough parameters, the light particle effective theory can mimic a more fundamental theory to any order in the Taylor expansion of its light particle amplitudes, when there is a genuine new particle of mass $M$ in the more fundamental theory this Taylor expansion has radius of convergence $M$. The light particle theory would then have to be discarded, at least for processes at and above threshold.

However it could in principle turn out, despite previous circumstantial evidence, that when the scale $\Lambda_{M}$ is reached there is no new particle at all, and the light particle theory can still be used there (albeit with limited predictivity) ${ }^{7}$; it is only possible, given the fact that all the low energy experimental data are in practice limited both in accuracy and quantity, to be certain of the existence of a new particle by actually producing it. Seeing is believing.

\section{Note Added.}

Recently two preprints have appeared [11] which also attempt to prove the decoupling theorem using the exact renormalization group. Both these authors work implicitly in the local limit $\Lambda_{0} \rightarrow \infty$, however, and unfortunately set artificial renormalization conditions on the heavy particle vertices at zero momentum, which renders the proof of decoupling relatively trivial, at the expense of substantially reducing its content.

7 It could be, for example, that the light particles are composite, as is the case is chiral perturbation theory. In this case too it would, at least in principle, be possible to compute the infinite number of coupling constants in the light particle effective theory in terms of those of some new theory of the constituents. 


\section{Acknowledgements.}

We would like to thank the Royal Society and the SERC for financial support during the period when most of this work was done, and the Theoretical Physics group of Oxford University for their hospitality; RST would also like to thank Jesus College, Oxford and the Leathersellers' Company for a graduate scholarship. 


\section{References}

[1] R. D. Ball and R. S. Thorne, OUTP-93-23P, CERN-TH.7067/93, to be published in Ann. Phys.

[2] K. G. Wilson and J. G. Kogut, Phys. Rep. 12C (1974) 75.

[3] J. Polchinski, Nucl. Phys. B231 (1984) 269.

[4] R. D. Ball and R. S. Thorne, CERN-TH.????/94, RAL-94-039.

[5] K. Symanzik, Comm. Math. Phys. 18 (1970) 7;

S. Weinberg, Phys. Rev. D8 (1973) 605;

T. Appelquist and J. Carazzone, Phys. Rev. D11 (1975) 2856.

[6] J. Ambjørn Comm. Math. Phys. 67 (1979) 109 ;

E. B. Manoukian, Jour. Math. Phys. 22 (1981) 572,2258;

T. Schimert and C. C. Chiang, Phys. Rev. D29 (1984) 241.

[7] C. Lee, Nucl. Phys. B161 (1979) 171;

Y. Kazama and Y. P. Yao, Phys. Rev. D21 (1980) 1116.

[8] H. Georgi, H. R. Quinn and S. Weinberg, Phys. Rev. Lett. 33 (1974) 451;

E. Witten, Nucl. Phys. B104 (1976) 445;

S. Weinberg, Phys. Lett. 91B (1980) 51;

B. A. Ovrut and H. J. Schnitzer, Phys. Rev. D21 (1980) 3369; D22 (1980) 2518; D24 (1981) 1695; Nucl. Phys. B184 (1981) 109;

H. Georgi, Nucl. Phys. B232 (1984) 61 ; B363 (1991) 301; (Proc. Suppl.) 29B,C (1992) 1.

[9] M. Veltman, Physica 29 (1963) 186.

[10] S. Weinberg, Physica 96A (1979) 327;

J. Gasser and H. Leutwyler, Ann. Phys. 158 (1984) 142; Nucl. Phys. B250 (1985) 465.

[11] L. Girardello and A. Zaffaroni, IFUM/432/FT, SISSA/14/94/EP;

C. Kim, SNUTP 94-20. 